\title{
A class of cyclotomic linear codes and their generalized Hamming weights
}

Fei Li

Received: date / Accepted: date

\begin{abstract}
Firstly, we give a formula on the generalized Hamming weight of linear codes constructed generically by defining sets. Secondly, by choosing properly the defining set we obtain a class of cyclotomic linear codes and then present two alternative formulas to calculate their generalized Hamming weights. Lastly, we determine their weight distribution and generalized Hamming weights partially. Especially, we solved the generalized Hamming weights completely in one case.
\end{abstract}

Keywords Cyclotomic linear code - Generalized Hamming weight · Weight distribution - Gauss sum · Gaussian period

Mathematics Subject Classification (2010) 94B05 $\cdot 11 \mathrm{~T} 22 \cdot 11 \mathrm{~T} 23$

\section{Introduction}

Let $q=p^{e}$ for a prime $p$. Denote $F_{Q}=F_{q^{m}}$ the finite field with $Q$ elements and $F_{q^{m}}^{*}$ the multiplicative group of $F_{q^{m}}$. We assume that $h$ is positive divisor of $Q-1$ and $1<h<\sqrt{Q}+1$. And $\theta$ is a fixed primitive element of $F_{q^{m}}$.

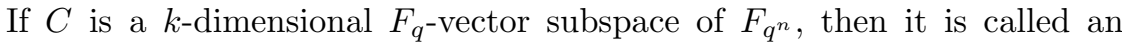
$[n, k, d]$ linear code with length $n$ and minimum Hamming distance $d$ over $F_{q}$. Denote $A_{i}$ the number of codewords with Hamming weight $i$ in $C$. If $\left|\left\{i: A_{i} \neq 0,1 \leq i \leq n\right\}\right|=t$, then $C$ is called a $t$-weight code. The readers are referred to [12] for more details and general theory of linear code.

This research is supported in part by National Natural Science Foundation of China (61602342).

Fei Li

E-mail: cczxlf@163.com

Faculty of School of Statistics and Applied Mathematics, Anhui University of Finance and Economics, Bengbu, Anhui Province, 233030, P.R.China 
A generic construction of linear code as below was proposed by Ding et al.([5], 6$]$. Let $D=\left\{d_{1}, d_{2}, \cdots, d_{n}\right\}$ be a subset of $F_{Q}^{*}$. Define a linear code $C_{D}$ of length $n$ over $F_{q}$ as following.

$$
C_{D}=\left\{\left(\operatorname{Tr}_{Q / q}\left(x d_{1}\right), \operatorname{Tr}_{Q / q}\left(x d_{2}\right), \ldots, T r_{Q / q}\left(x d_{n}\right)\right): x \in F_{Q}\right\}
$$

and $D$ is called the defining set. The method is used in lots of research to get linear codes with few weights $[9,17,23,24$ by choosing properly defining sets.

For an $[n, k, d]$ linear code $C$, we could extend Hamming weight to obtain the concept of the generalized Hamming weight(GHW) $d_{r}(C)(0<r \leq k)$ (see $[15,20)$. It is defined as follows. Denote $[C, r]_{q}$ the set of the $r$-dimensional $F_{q}$-vector subspace of $C$. For $V \in[C, r]_{q}$, let $\operatorname{Supp}(V)$ be the set of positions $i$ where there exists a codeword $x=\left(x_{1}, x_{2}, \cdots, x_{n}\right) \in V$ with $x_{i} \neq 0$. Then the $r$-th generalized Hamming weight $(\mathrm{GHW}) d_{r}(C)$ of linear code $C$ is defined by

$$
d_{r}(C)=\min \left\{|\operatorname{Supp}(V)|: V \in[C, r]_{q}\right\},
$$

and $\left\{d_{i}(C): 1 \leq i \leq k\right\}$ is defined as the weight hierarchy of $C$. In particular, the GHW $d_{1}(C)$ is just the usual Hamming weight $d$. Since the classic results of Wei in the paper 20] in 1991, lots of authors paid attention to the generalized Hamming weight. In 19, the readers can find a survey on the results up to 1995 about GHW. Afterwards there have been a number of studies on generalized Hamming weight about some particular families of codes $1,2,3,7,11,13,14,21$, 22 . It is worth mentioning that the recent work in 22 gave a very instructive approach to calculate the GHWs of irreducible cyclic codes. Generally, it is not easy to determine the weight hierarchy.

The rest of this paper is organised as follows: in Section 2, we review basic concepts and results on Gauss sum and Exponential sum useful for this paper; in Section 3, we follow the work of Cunsheng Ding et al. 10,8 to construct a class of cyclotomic linear codes and give general formulas on $d_{r}(C)$. Meanwhile, we determine their weight distribution under certain conditions; in Section 4, we give the conclusion of this paper.

\section{Preliminaries}

We start with the additive character. Let $b \in F_{Q}$, the mapping

$$
\chi_{b}(c)=\zeta_{p}^{T r_{Q / p}(b c)} \text { for all } c \in F_{Q}
$$

defines an additive character of $F_{Q}$, where $\zeta_{p}=e^{\frac{2 \pi \sqrt{-1}}{p}}$ and $\operatorname{Tr}_{Q / p}$ is the trace function from $F_{Q}$ to $F_{p}$. Particularly, the character $\chi_{1}$ is called the canonical additive character of $F_{Q}$. The multiplicative characters of $F_{Q}$ are defined by

$$
\psi_{j}\left(\theta^{k}\right)=e^{2 \pi \sqrt{-1} j k /(Q-1)} \text { for } k=0,1, \ldots, Q-2,0 \leq j \leq Q-2 .
$$


For each additive $\chi$ and multiplicative character $\psi$, we define Gauss sums $G_{Q}(\psi, \chi)$ over $F_{Q}$ by

$$
G_{Q}(\psi, \chi)=\sum_{x \in F_{Q}^{*}} \psi(x) \chi(x) .
$$

The readers can refer to [16] for more information about the explicit values of Gauss sums.

For each $\alpha \in F_{Q}$, a Exponential sum $S(\alpha)$ is defined as follows.

$$
S(\alpha)=\sum_{x \in F_{Q}} \chi_{1}\left(\alpha x^{h}\right)
$$

For a integer $i$, define

$$
C_{i}=\left\{\theta^{i}\left(\theta^{h}\right)^{j}: 0 \leq j<\frac{Q-1}{h}\right\}, \eta_{i}=\sum_{x \in C_{i}} \chi_{1}(x) .
$$

It is easy to see $C_{u}=C_{v}$ if and only if $u \equiv v(\bmod h)$. These sets $C_{i}$ and numbers $\eta_{i}$ are called the cyclotomic classes and Gaussian periods (see [4]) of order $h$ in $F_{Q}^{*}$, respectively. By definition, it is not hard to get $S\left(\theta^{i}\right)=h \eta_{i}+1$.

The following lemma is about the explicit values of the Exponential sum $S(\alpha)$. It will be used later.

Lemma 1.([18]) Assume $m=2 l k, h \mid\left(q^{k}+1\right)$. Then for any $\alpha \in F_{Q}^{*}$,

$$
S(\alpha)= \begin{cases}(-1)^{l} \sqrt{Q}, & \text { if } \alpha \notin C_{h_{0}}, \\ (-1)^{l-1}(h-1) \sqrt{Q}, & \text { if } \quad \alpha \in C_{h_{0}},\end{cases}
$$

where

$$
h_{0}=\left\{\begin{array}{l}
\frac{h}{2}, \text { if } p>2, l \text { odd, and } \frac{q^{k}+1}{h} \text { odd }, \\
0, \text { otherwise } .
\end{array}\right.
$$

Here we present three bounds on GHWs of linear codes. The readers may refer to the literature [19] for them.

Lemma 2. Let $C$ be a linear code over $F_{q}$ with parameters $[\mathrm{n}, \mathrm{m}]$. For $1 \leq r \leq m$,

1. (Singleton type bound) $r \leq d_{r}(C) \leq n-m+r$. And $C$ is called an $r$-MDS code if $d_{r}(C)=n-m+r$.

2. (Griesmer-like bound)

$$
d_{r}(C) \geq \sum_{i=0}^{r-1}\left\lceil\frac{d_{r}(C)}{q^{i}}\right\rceil
$$

3. (Plotkin-like bound)

$$
d_{r}(C) \leq \sum_{i=0}^{r-1}\left\lfloor\frac{n\left(q^{r}-1\right) q^{m-r}}{q^{m}-1}\right\rfloor
$$




\section{Main Results and Proofs}

First of all, we give a general formula to compute the GHW of the linear code defined by the generic method in (1) with the defining set $D$.

Theorem 1. For each $r(1 \leq r \leq m)$, if the dimension of $C_{D}$ is $m$, then $d_{r}\left(C_{D}\right)=n-\max \left\{|D \bigcap H|: H \in\left[F_{Q}, m-r\right]_{q}\right\}$.

Proof. The proof is similar to that of Theorem 6 in 22. But for the convenience of readers, we provide the proof. Let $\phi$ be such a mapping from $F_{Q}$ to $F_{q}^{n}$ that

$$
\phi(x)=\left(\operatorname{Tr}_{Q / q}\left(x d_{1}\right), \operatorname{Tr}_{Q / q}\left(x d_{2}\right), \ldots, \operatorname{Tr}_{Q / q}\left(x d_{n}\right)\right)
$$

for each $x \in F_{Q}$. Obviously, $\phi$ is a $F_{q}$-linear mapping and the image of $\phi$ is $C_{D}$. And $\phi$ is injective since the dimension of $C_{D}$ is $m$. For a $r$-dimension subspace $C_{r} \in\left[C_{D}, r\right]_{q}$, denote $H_{r}$ the pre-image $\phi^{-1}\left(C_{r}\right)$ in $F_{Q}$. Also $H_{r}$ is a $r$-dimension subspace of $F_{Q}$. By definition, $d_{r}\left(C_{D}\right)=n-\max \left\{N\left(C_{r}\right): C_{r} \in\right.$ $\left.\left[C_{D}, r\right]_{q}\right\}$, where

$$
\begin{aligned}
N\left(C_{r}\right) & =\sharp\left\{i: 1 \leq i \leq n, c_{i}=0 \text { for each } c=\left(c_{1}, c_{2}, \ldots, c_{n}\right) \in C_{r}\right\} \\
& =\sharp\left\{i: 1 \leq i \leq n, \operatorname{Tr}_{Q / q}\left(\beta d_{i}\right)=0 \text { for each } \beta \in H_{r}\right\}
\end{aligned}
$$

Let $\left\{\beta_{1}, \beta_{2}, \ldots, \beta_{r}\right\}$ be an $F_{q}$-basis of $H_{r}$. Hence

$$
\begin{gathered}
N\left(C_{r}\right)=\frac{1}{q^{r}} \sum_{u_{i} \in \bar{D}}\left(\sum_{x_{1} \in F_{q}} \zeta_{p}^{T r_{q / p}\left(T r_{Q / q}\left(\beta_{1} u_{i}\right) x_{1}\right)}\right) \ldots\left(\sum_{x_{r} \in F_{q}} \zeta_{p}^{T r_{q / p}\left(T r_{Q / q}\left(\beta_{r} u_{i}\right) x_{r}\right)}\right) \\
\quad=\frac{1}{q^{r}} \sum_{\beta \in H_{r}} \sum_{i=1}^{n} \zeta_{p}^{T r_{Q / p}\left(\beta d_{i}\right)}=\frac{1}{q^{r}} \sum_{i=1}^{n} \sum_{\beta \in H_{r}} \zeta_{p}^{T r_{Q / p}\left(\beta d_{i}\right)}
\end{gathered}
$$

Let $H^{\perp}$ be the dual of $H$ defined by $H^{\perp}=\left\{v \in F_{Q}: \operatorname{Tr}_{Q / q}(u v)=0, u \in H\right\}$. We know that $\operatorname{dim}_{F_{q}}(H)+\operatorname{dim}_{F_{q}}\left(H^{\perp}\right)=m$.

For $y \in F_{Q}$,

$$
\sum_{\beta \in H_{r}} \zeta_{p}^{T r_{Q / p}(\beta y)}= \begin{cases}\left|H_{r}\right|, & \text { if } y \in H_{r}^{\perp}, \\ 0, & \text { otherwise } .\end{cases}
$$

By the above equation, we have

$$
N\left(C_{r}\right)=\frac{1}{q^{r}} \sum_{y \in D \bigcap H_{r}^{\perp}}\left|H_{r}\right|=\left|D \bigcap H_{r}^{\perp}\right| .
$$

So the desired result follows from that there is a bijection between $\left[F_{Q}, r\right]_{q}$ and $\left[F_{Q}, m-r\right]_{q}$. We complete the proof.

From now on, we suppose $h(q-1)$ is also a divisor of $Q-1$. We construct linear codes by choosing the defining set to be

$$
\bar{D}=\left\{\theta^{t_{1}} d_{1}, \ldots, \theta^{t_{1}} d_{n_{0}}, \theta^{t_{2}} d_{1}, \ldots, \theta^{t_{2}} d_{n_{0}}, \cdots, \theta^{t_{s}} d_{1}, \ldots, \theta^{t_{s}} d_{n_{0}}\right\}
$$


$d_{i}=\theta^{h(i-1)}, n_{0}=\frac{q^{m}-1}{h(q-1)}, 0 \leq t_{1}<t_{2}<\ldots<t_{s} \leq h-1,1 \leq s \leq h$. Thus we obtain a class of cyclotomic linear codes $C_{\bar{D}}$ since $\bar{D}$ is closely related to the cyclotomic classes of order $h$ in $F_{Q}^{*}$.

In addition to Theorem 1, we give alternative formulas to calculate the GHW of cyclotomic linear codes $C_{\bar{D}}$.

Theorem 2. For each $r, 1 \leq r \leq m, d_{r}\left(C_{\bar{D}}\right)=s n_{0}-N_{r}$, where

$$
\begin{gathered}
\text { (1) } N_{r}=\frac{s\left(q^{m}-q^{r}\right)}{h q^{r}(q-1)}+\frac{1}{h q^{r}(q-1)} \max \left\{A_{H_{r}}: H_{r} \in\left[F_{Q}, r\right]_{q}\right\}, \\
A_{H_{r}}=\sum_{j=1}^{s} \sum_{\lambda=1}^{h-1} \sum_{\beta \in H_{r}^{*}} \overline{\varphi^{\lambda}}\left(\beta \theta^{t_{j}}\right) G_{Q}\left(\varphi^{\lambda}\right)
\end{gathered}
$$

or

(2) $N_{r}=\frac{s n_{0}}{q^{r}}+\frac{1}{q^{r}(q-1)} \max \left\{\sum_{i=0}^{h-1}\left|H_{r} \bigcap\left(\bigcup_{j=1}^{s} C_{i-t_{j}}\right)\right| \eta_{i}: H_{r} \in\left[F_{Q}, r\right]_{q}\right\}$

Proof. (1) By definition, $d_{r}\left(C_{\bar{D}}\right)=s n_{0}-N_{r}, N_{r}=\max \left\{N\left(C_{r}\right): C_{r} \in\right.$ $\left.\left[C_{\bar{D}}, r\right]_{q}\right\}$. Let $\left\{\beta_{1}, \beta_{2}, \ldots, \beta_{r}\right\}$ be an $F_{q}$-basis of $H_{r}$. Here $\phi\left(H_{r}\right)=C_{r}$. See the proof of Theorem 1 for the definitions of $N\left(C_{r}\right)$ and $\phi$. Set $H_{r}^{*}=H_{r} \backslash\{0\}$. Hence

$$
\begin{gathered}
N\left(C_{r}\right)=\frac{1}{q^{r}} \sum_{u_{i} \in \bar{D}}\left(\sum_{x_{1} \in F_{q}} \zeta_{p}^{T r_{q / p}\left(T r_{Q / q}\left(\beta_{1} u_{i}\right) x_{1}\right)}\right) \ldots\left(\sum_{x_{r} \in F_{q}} \zeta_{p}^{T r_{q / p}\left(T r_{Q / q}\left(\beta_{r} u_{i}\right) x_{r}\right)}\right) \\
=\frac{1}{q^{r}} \sum_{\beta \in H_{r}} \sum_{u_{i} \in \bar{D}} \zeta_{p}^{T r_{Q / p}\left(\beta u_{i}\right)}=\frac{s n_{0}}{q^{r}}+\frac{1}{q^{r}} \sum_{\beta \in H_{r}^{*}} \sum_{u_{i} \in \bar{D}} \zeta_{p}^{T r_{Q / p}\left(\beta u_{i}\right)} \\
=\frac{s n_{0}}{q^{r}}+\frac{1}{q^{r}(q-1)} \sum_{\beta \in H_{r}^{*}} \sum_{u_{i} \in F_{q}^{*} \bar{D}} \zeta_{p}^{T r_{Q / p}\left(\beta u_{i}\right)} \\
=\frac{s n_{0}}{q^{r}}+\frac{1}{h q^{r}(q-1)} \sum_{j=1}^{s} \sum_{\beta \in H_{r}^{*}} \sum_{x \in F_{Q}^{*}} \zeta_{p}^{T r_{Q / p}(\beta x)} \sum_{\lambda=0}^{h-1} \varphi^{\lambda}\left(\theta^{-t_{j}} x\right) \\
=\frac{s\left(q^{m}-q^{r}\right)}{h q^{r}(q-1)}+\frac{1}{h q^{r}(q-1)} \sum_{j=1}^{s} \sum_{\lambda=1}^{h-1} \sum_{\beta \in H_{r}^{*}} \sum_{x \in F_{Q}^{*}} \zeta_{p}^{T r_{Q / p}(\beta x)} \varphi^{\lambda}\left(\theta^{-t_{j}} x\right) \\
N\left(C_{r}\right)=\frac{s\left(q^{m}-q^{r}\right)}{h q^{r}(q-1)}+\frac{1}{h q^{r}(q-1)} \sum_{j=1}^{s} \sum_{\lambda=1}^{h-1} G_{Q}\left(\varphi^{\lambda}\right) \sum_{\beta \in H_{r}^{*}} \frac{1}{\varphi^{\lambda}}\left(\theta^{t_{j}} \beta\right) \\
N\left(C_{r}\right)=\frac{s\left(q^{m}-q^{r}\right)}{h q^{r}(q-1)}+\frac{1}{h q^{r}(q-1)} \sum_{j=1}^{s} \sum_{\lambda=1}^{h-1} \frac{\varphi^{\lambda}}{\lambda}\left(\theta^{t_{j}}\right) G_{Q}\left(\varphi^{\lambda}\right) \sum_{\beta \in H_{r}^{*}} \frac{\varphi^{\lambda}}{}(\beta)
\end{gathered}
$$


For simplicity, we set $A_{H_{r}}=\sum_{j=1}^{s} \sum_{\lambda=1}^{h-1} \overline{\varphi^{\lambda}}\left(\theta^{t_{j}}\right) G_{Q}\left(\varphi^{\lambda}\right) \sum_{\beta \in H_{r}^{*}} \overline{\varphi^{\lambda}}(\beta)$. So

$$
N\left(C_{r}\right)=\frac{s\left(q^{m}-q^{r}\right)}{h q^{r}(q-1)}+\frac{A_{H_{r}}}{h q^{r}(q-1)} .
$$

(2) By the proof of Part (1), we have

$$
\begin{gathered}
N\left(C_{r}\right)=\frac{s n_{0}}{q^{r}}+\frac{1}{q^{r}(q-1)} \sum_{\beta \in H_{r}^{*}} \sum_{u \in F_{q}^{*} \bar{D}} \zeta_{p}^{T r_{Q / p}(\beta u)} \\
=\frac{s n_{0}}{q^{r}}+\frac{1}{q^{r}(q-1)} \sum_{\beta \in H_{r}^{*}} \sum_{j=1}^{s} \sum_{u \in C_{t_{j}}} \zeta_{p}^{T r_{Q / p}(\beta u)} \\
=\frac{s n_{0}}{q^{r}}+\frac{1}{q^{r}(q-1)} \sum_{j=1}^{s} \sum_{\beta \in H_{r}^{*}} \sum_{u \in C_{t_{j}}} \chi_{1}(\beta u) .
\end{gathered}
$$

By definition, $\eta_{i}=\sum_{x \in C_{i}} \chi_{1}(x)$. So

$$
\sum_{j=1}^{s} \sum_{\beta \in H_{r}^{*}} \sum_{u \in C_{t_{j}}} \chi_{1}(\beta u)=\sum_{i=0}^{h-1} \sum_{j=1}^{s}\left|H_{r} \bigcap C_{i-t_{j}}\right| \eta_{i}=\sum_{i=0}^{h-1}\left|H_{r} \bigcap\left(\bigcup_{j=1}^{s} C_{i-t_{j}}\right)\right| \eta_{i}
$$

Then the desired result follows and the proof is completed.

\section{Remarks.}

(1) If $s=h$, then by Theorem 1 or Theorem 3(2), it is easy to get $d_{r}\left(C_{\bar{D}}\right)=\frac{q^{m}-q^{m-r}}{q-1}$.

(2) Also by Theorem 1 or Theorem 2(2), it is easy to get $d_{r}\left(C_{\bar{D}}\right)=$ $\frac{s\left(q^{m}-1\right)}{h(q-1)}-m+r$ if $r=m-1$. So by Singleton type bound in Lemma $2, C_{\bar{D}}$ is an $(m-1)$-MDS code [19] over $F_{q}$. Especially, if $m=2$, then the code $C_{\bar{D}}$ is an $\left[\frac{s(q+1)}{h}, 2, \frac{s(q+1)}{h}-1\right]$ MDS code [12] over $F_{q}$.

(3) Generally, it is difficult to establish linkages between the additive properties and the multiplicative ones of a field. So Theorem 1 and 2 indicate it is difficult to give the explicit values of the generalized Hamming weight of $C_{\bar{D}}$ for other cases.

Next under certain conditions, we give the weight distribution of cyclotomic linear codes $C_{\bar{D}}$ in the following theorem.

Theorem 3. Assume $m=2 l k$ and $h \mid\left(q^{k}+1\right)$. Then the code $C_{\bar{D}}$ is an $\left[\frac{s(Q-1)}{h(q-1)}, m\right]$ linear code over $F_{q}$ with weight distribution in table 1 . And the dual code $C_{\bar{D}}^{\perp}$ of $C_{\bar{D}}$ is an $\left[\frac{s(Q-1)}{h(q-1)}, \frac{s(Q-1)}{h(q-1)}-m, d^{\perp}\right]$ linear code with minimum distance $d^{\perp} \geq 3$.

Proof. For $x \in F_{q}^{*}$, we have

$$
w\left(c_{x}\right)=s n_{0}-\sum_{j=1}^{s}\left|\left\{i: 1 \leq i \leq n_{0}, \operatorname{Tr}_{Q / q}\left(x \theta^{t_{j}} d_{i}\right)=0\right\}\right|
$$


Table 1 The weight distribution of the codes of Theorem 3 .

$$
\begin{aligned}
& \begin{array}{|c|c|}
\hline \text { Weight } w & \text { Multiplicity } A \\
\hline 0 & 1 \\
\hline \frac{1}{q h}\left(s(Q-1)+s+(-1)^{l}(h-s) \sqrt{Q}\right) & \frac{s(Q-1)}{h} \\
\hline \frac{1}{q h}\left(s(Q-1)+s-s(-1)^{l} \sqrt{Q}\right) & \frac{(h-s)(Q-1)}{h} \\
\hline
\end{array} \\
& =s n_{0}-\frac{1}{q} \sum_{j=1}^{s} \sum_{i=1}^{n_{0}} \sum_{s \in F_{q}} \zeta_{p}^{T r_{q / p}\left(s T r_{Q / q}\left(x \theta^{t_{j}} d_{i}\right)\right)} \\
& =\frac{s(Q-1)}{h q}-\frac{1}{q} \sum_{j=1}^{s} \sum_{i=1}^{n_{0}} \sum_{s \in F_{q}^{*}} \chi_{1}\left(s x \theta^{t_{j}} d_{i}\right) \\
& =\frac{s(Q-1)}{h q}-\frac{1}{q} \sum_{j=1}^{s} \sum_{k=1}^{\frac{Q-1}{h}} \chi_{1}\left(x \theta^{t_{j}+h k}\right)=\frac{s(Q-1)}{h q}-\frac{1}{q h} \sum_{j=1}^{s} \sum_{k=1}^{Q-1} \chi_{1}\left(x \theta^{t_{j}+h k}\right) \\
& =\frac{s(Q-1)}{h q}-\frac{1}{q h} \sum_{j=1}^{s}\left(\left(S\left(x \theta^{t_{j}}\right)-1\right)=\frac{1}{q h}\left(s(Q-1)+s-\sum_{j=1}^{s} S\left(x \theta^{t_{j}}\right)\right)\right.
\end{aligned}
$$

By Lemma 1, we have

$$
w\left(c_{x}\right)= \begin{cases}\frac{1}{q h}\left(s(Q-1)+s+(-1)^{l}(h-s) \sqrt{Q}\right), & \text { if one of } x \theta^{t_{j}} \in C_{h_{0}}, \\ \frac{1}{q h}\left(s(Q-1)+s-s(-1)^{l} \sqrt{Q}\right), & \text { otherwise. }\end{cases}
$$

As for the parameters of the dual code, it is enough to prove $d^{\perp} \geq 3$. It is easy to show that any two elements in $\bar{D}$ are linearly independent over $F_{q}$. Then the desired results follow and we complete the proof.

Corollary 1. Assume $m=2 l k$ and $h \mid\left(q^{k}+1\right)$. If $(l, 2)=1$, then

$$
d_{r}\left(C_{\bar{D}}\right)= \begin{cases}\frac{s\left(q^{m}-q^{m-r}\right)+(s-h) q^{\frac{m}{2}-r}\left(q^{r}-1\right)}{h(q-1)}, & \text { if } 1 \leq r \leq \frac{m}{2} \\ \frac{s\left(q^{m}-1\right)-h\left(q^{m-r}-1\right)}{h(q-1)}, & \text { if } \frac{m}{2} \leq r \leq m\end{cases}
$$

Proof. By Lemma 1, we have $\eta_{i}=\frac{(h-1) \sqrt{Q}-1}{h}$ if $i=h_{0}$, otherwise $\eta_{i}=\frac{-\sqrt{Q}-1}{h}$. So by Theorem 3(2), we get $\sum_{j=1}^{s} \sum_{i=0}^{h-1}\left|H_{r} \bigcap C_{i-t_{j}}\right| \eta_{i}$

$$
\begin{aligned}
= & \sum_{j=1}^{s} \sum_{i=0}^{h-1}\left|H_{r} \bigcap C_{i-t_{j}}\right| \frac{-\sqrt{Q}-1}{h}+\sum_{j=1}^{s}\left|H_{r} \bigcap C_{h_{0}-t_{j}}\right|\left(\eta_{h_{0}}-\frac{-\sqrt{Q}-1}{h}\right) . \\
& \sum_{j=1}^{s} \sum_{i=0}^{h-1}\left|H_{r} \bigcap C_{i-t_{j}}\right| \eta_{i}=s\left(q^{r}-1\right) \frac{-\sqrt{Q}-1}{h}+\sqrt{Q} \sum_{j=1}^{s}\left|H_{r} \bigcap C_{h_{0}-t_{j}}\right|
\end{aligned}
$$


If $(l, 2)=1$, then $F_{q^{l k}} \subset C_{0}$. Notice that $C_{i}=\theta^{i} C_{0}$ and $\theta^{i} H_{r}$ is also a $r$-dimension subspace. So we have

$$
\max \left\{\left|H_{r} \bigcap\left(\bigcup_{i=0}^{h-1} C_{h_{0}-t_{j}}\right)\right|: H_{r} \in\left[F_{Q}, r\right]_{q}\right\}=q^{r}-1
$$

for each $r$ with $1 \leq r \leq \frac{m}{2}$. By Theorem 3, we get the first part of the corollary. If $\frac{m}{2} \leq r \leq m$, then $0 \leq m-r \leq \frac{m}{2}$ and $\max \left\{|\bar{D} \cap H|: H \in\left[F_{Q}, m-r\right]_{q}\right\}=$ $\frac{q^{m-r}-1}{q-1}$. By Theorem 1, we get the second part of this corollary. The proof is completed.

Corollary 2. Also assume $m=2 l k$ and $h \mid\left(q^{k}+1\right)$. If $l=2^{u} l^{\prime}$ with $u>0,\left(l^{\prime}, 2\right)=1$, and $s<h$, then

$$
d_{r}\left(C_{\bar{D}}\right)= \begin{cases}\frac{s q^{\frac{m}{2}-r}\left(q^{r}-1\right)\left(q^{\frac{m}{2}}-1\right)}{h(q-1)}, \text { if } 1 \leq r \leq l^{\prime} k, \\ \frac{s\left(q^{m}-1\right)-h\left(q^{m-r}-1\right)}{h(q-1)}, \text { if } m-l^{\prime} k \leq r \leq m .\end{cases}
$$

Proof. By assumption, we have $F_{q^{l^{\prime} k}} \subset C_{0}$. The remaining proof is similar to that of Corollary 1. We omit the details.

\section{Concluding Remarks}

In this paper, we give a formula to compute the generalized Hamming weight of linear code $C_{D}$, which is constructed by the generic method proposed by Ding et al. By choosing properly the defining set, we present a class of cyclotomic linear codes $C_{\bar{D}}$. We give two alternative formulas about their generalized Hamming weights by Gauss sums and Gaussian periods. Under certain conditions, we solve the weight distribution of $C_{\bar{D}}$ and find it is a two-weight linear code. We determine completely the generalized Hamming weigh of $C_{\bar{D}}$ in one case.

\section{References}

1. M. Bras-Amors, K. Lee, and A. Vico-Oton, New lower bounds on the generalized Hamming weights of AG codes, IEEE Trans. Inf. Theory, 60(10), 5930-5937(2014).

2. A. I. Barbero and C. Munuera, The weight hierarchy of Hermitian codes, SIAM J. Discrete Math., 13(1), 79-104(2000).

3. J. Cheng and C.-C. Chao, On generalized Hamming weights of binary primitive BCH codes with minimum distance one less than a power of two, IEEE Trans. Inf. Theory, 43(1), 294-298(1997).

4. C. Ding, Codes from Difference Sets, World Scientific, Singapore(2015).

5. C. Ding, Linear codes from some 2-designs, IEEE Trans. Inf. Theory, 61(6), 32653275(2015).

6. K. Ding, C. Ding, Bianry linear codes with three weights, IEEE Communication Letters, 18(11), 1879-1882(2014).

7. M. Delgado, J. I. Farrn, P. A. Garca-Snchez, and D. Llena, On the weight hierarchy of codes coming from semigroups with two generators, IEEE Trans. Inf. Theory, 60(1), $282-295(2014)$ 
8. C. Ding, J. Luo, H. Niederreiter, Two-weight codes punctured from irreducible cyclic codes, in: Y. Li, et al. (Eds.), Proceedings of the First Worshop on Coding and Cryptography, World Scientific, Singapore, pp. 119-124, 2008.

9. C. Ding, C. Li, N.Li, Z. Zhou, Three-weight cyclic codes and their weight distributions. Discret. Math., 339(2), 415-427 (2016).

10. C. Ding and H. Niederreiter, Cyclotomic linear codes of order 3, IEEE Trans. Inf. Theory, 53(6), 2274-2277(2007).

11. P. Heijnen and R. Pellikaan, Generalized Hamming weights of q-ary ReedCMuller codes, IEEE Trans. Inf. Theory, 44(1), 181-196(1998).

12. W. C. Huffman and V. Pless, Fundamentals of error-correcting codes, Cambridge University Press, Cambridge(2003).

13. G. Jian, R. Feng and H. Wu, Generalized Hamming weights of three classes of linear codes, Finite Fields and Their Applications, 45, 341-354(2017).

14. H. Janwa and A. K. Lal, On the generalized Hamming weights of cyclic codes, IEEE Trans. Inf. Theory, 43(1), 299-308(1997).

15. T. Kl $\phi$ ve, The weight distribution of linear codes over $G F\left(q^{l}\right)$ having generator matrix over $G F(q>)$, Discrete Math., 23(2), 159-168(1978).

16. R. Lidl, H. Niederreiter, Finite fields, Cambridge University Press, New York(1997).

17. C. Li, Q. Yue, and F. Li, Hamming weights of the duals of cyclic codes with two zeros, IEEE Trans. Inf. Theory, 60(7), 3895-3902(2014).

18. M.J. Moisio, A note on evaluations of some exponential sums, Acta Arith., 93, 117$119(2000)$

19. M. A. Tsfasman, S. G. Vladut, Geometric approach to higher weights, IEEE Trans. Inf. Theory, 41(6), 1564-1588(1995).

20. V.K.Wei, Generalized Hamming weights for linear codes, IEEE Trans. Inf. Theory, 37(5), 1412-1418(1991).

21. M. Xiong, S. Li, and G. Ge. The weight hierarchy of some reducible cyclic codes. IEEE Trans. Inform. Theory, 62(7), 4071-4080(2016).

22. M. Yang, J. Li, K. Feng and D. Lin, Generalized Hamming weights of irreducible cyclic codes, IEEE Trans. Inf. Theory, 61(9), 4905-4913(2015).

23. S.Yang, Z.Yao, Complete weight enumerators of a family of three-weight linear codes. Des. Codes Cryptogr. 82(3), 663-674(2017).

24. Z. Zhou, N. Li, C. Fan and T. Helleseth, Linear codes with two or three weights from quadratic bent functions, Des. Codes Cryptogr., 81(2), 283-295(2016). 